\title{
Tetra- and pentaquark spectroscopy
}

\section{E. Spadaro Norella ${ }^{a, b, *}$ on behalf of LHCb collaboration}

${ }^{a}$ Università degli Studi di Milano

${ }^{b}$ INFN Sezione di Milano,

via Celoria 16, Milan, Italy

E-mail: elisabetta.spadaro.norella@cern.ch

Recent results on exotic spectroscopy from the LHCb experiment are introduced in these proceedings. They contain the evidence for a new pentaquark candidate decaying to $J / \psi p$ and $J / \psi \bar{p}$ in the $B_{s}^{0} \rightarrow J / \psi p \bar{p}$ decays and the observation of four new resonances decaying to $J / \psi K^{+}$and $J / \psi \phi$ in the $B^{+} \rightarrow J / \psi \phi K^{+}$decays, two of which are tetraquarks with strangeness.

The Ninth Annual Conference on Large Hadron Collider Physics - LHCP2021

7-12 June 2021

Online

${ }^{*}$ Speaker 


\section{Introduction}

Tetraquark and pentaquark states, also called exotic states, are hadrons with a more complex internal structure than the standard mesons and baryons and are composed by four and five quarks, respectively. Although they have been originally proposed by Gell-Mann [1] and Zweig [2] in the quark model, their first evidence was confirmed only in 2003 with the first tetraquark candidate, the $X$ (3872), observed by the Belle experiment in the $J / \psi \pi^{+} \pi^{-}$mass spectrum [3]. The field of exotic spectroscopy has gained an increasing interest especially after the observation of pentaquark states, made by $\mathrm{LHCb}$ in 2015 in the $\Lambda_{b}^{0} \rightarrow J / \psi p K^{-}$decays [4]. More than thirty exotic states have been seen over the last few years. Recent results on exotic states from the LHCb experiment are reported in these proceedings.

\section{Evidence for a new state decaying to $J / \psi p$ and $J / \psi \bar{p}$ system}

The first observation of pentaquark states, $P_{c}(4380)^{+}$and $P_{c}(4450)^{+}$, was reported by the LHCb experiment in 2015 in the analysis of $\Lambda_{b}^{0} \rightarrow J / \psi p K^{-}$decays [4]. In 2019, with an updated analysis performed with the full $9 \mathrm{fb}^{-1}$ LHCb dataset, another state at mass $4312 \mathrm{MeV}$ was observed and the peak at mass $4450 \mathrm{MeV}$ was resolved into two separate peaks [5]. The $B_{s}^{0} \rightarrow J / \psi p \bar{p}$ decay, first observed by the LHCb experiment in 2019 [6], may be sensitive to the resonant $P_{c}$ structures $[4,5]$ decaying to $J / \psi p$ and $J / \psi \bar{p}$ final states and to the glueball $f_{J}(2220)$ predicted in Ref. [7]. The analysis of this mode is based on around 800 signal events collected by LHCb and corresponding to $9 \mathrm{fb}^{-1}$ of integrated luminosity [8]. The selection has been optimised with multivariate techniques exploiting the information of the particle identification variables of the protons to distinguish between signal and background candidates. The fit to the $J / \psi p \bar{p}$ invariant mass is shown in Fig. 1. In order to investigate the resonant structure of this decay, a four-dimensional amplitude analysis of the flavour untagged $B_{s}^{0}$ sample is employed, using the helicity formalism [9] and assuming $C P$ symmetry. The fit parameters are extracted from an unbinned maximum likelihood fit to the sum of the signal and the background components. The best model describing the data contains a non-resonant contribution in the $p \bar{p}$ decay chain plus two $P_{c}$ resonances, $P_{c}^{+}$and $P_{c}^{-}$, decaying to $J / \psi p$ and $J / \psi \bar{p}$, with mass and width fixed to the same values. These states are referred to as a single $P_{c}$ state. The results of the fit are shown in Fig. 2, projected onto the phase-space variables, $\left(m_{p \bar{p}}, \cos \theta_{\mu}, \cos \theta_{p}, \varphi\right)$, and the $m(J / \psi p)$ and $m(J / \psi \bar{p})$ invariant masses. Different $J^{P}$ hypothesis for the $P_{c}$ state, i.e. $1 / 2^{ \pm}, 3 / 2^{ \pm}$, have been investigated but none of them can be excluded at the $90 \%$ of confidence level (CL). The mass and width of this $P_{c}$ state are not compatible with the previously observed pentaquark states and are measured to be $M\left(P_{c}\right)=4337_{-4}^{+7}$ (stat $)_{-2}^{+2}$ (syst) MeV and $\Gamma\left(P_{c}\right)=29_{-12}^{+26}$ (stat) ${ }_{-14}^{+14}$ (syst) $\mathrm{MeV}$, respectively. Its significance is estimated with a frequentist approach to lie in the range of 3.1 and $3.7 \sigma$ depending on the $J^{P}$ hypothesis considered, providing evidence for a new pentaquark-like state. With this analysis, no evidence is seen for either the $P_{c}$ (4312) state [5] or the glueball $f_{J}(2220)$ [7].

\section{Observation of new resonances decaying to $J / \psi K^{+}$and $J / \psi \phi$}

The $B^{+} \rightarrow J / \psi \phi K^{+}$mode has been previously studied by LHCb using the Run 1 dataset corresponding to $3 \mathrm{fb}^{-1}$ [10]. Here, the results of the updated analysis performed with the full 


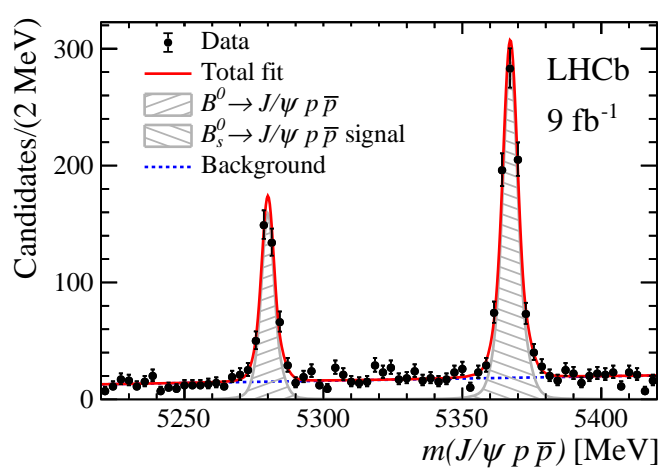

Figure 1: Fit to the $m(J / \psi p \bar{p})$ invariant mass, where the $B_{s}^{0}$ signal is modelled by two Crystal Ball functions and the combinatorial background by a first-order polynomial.
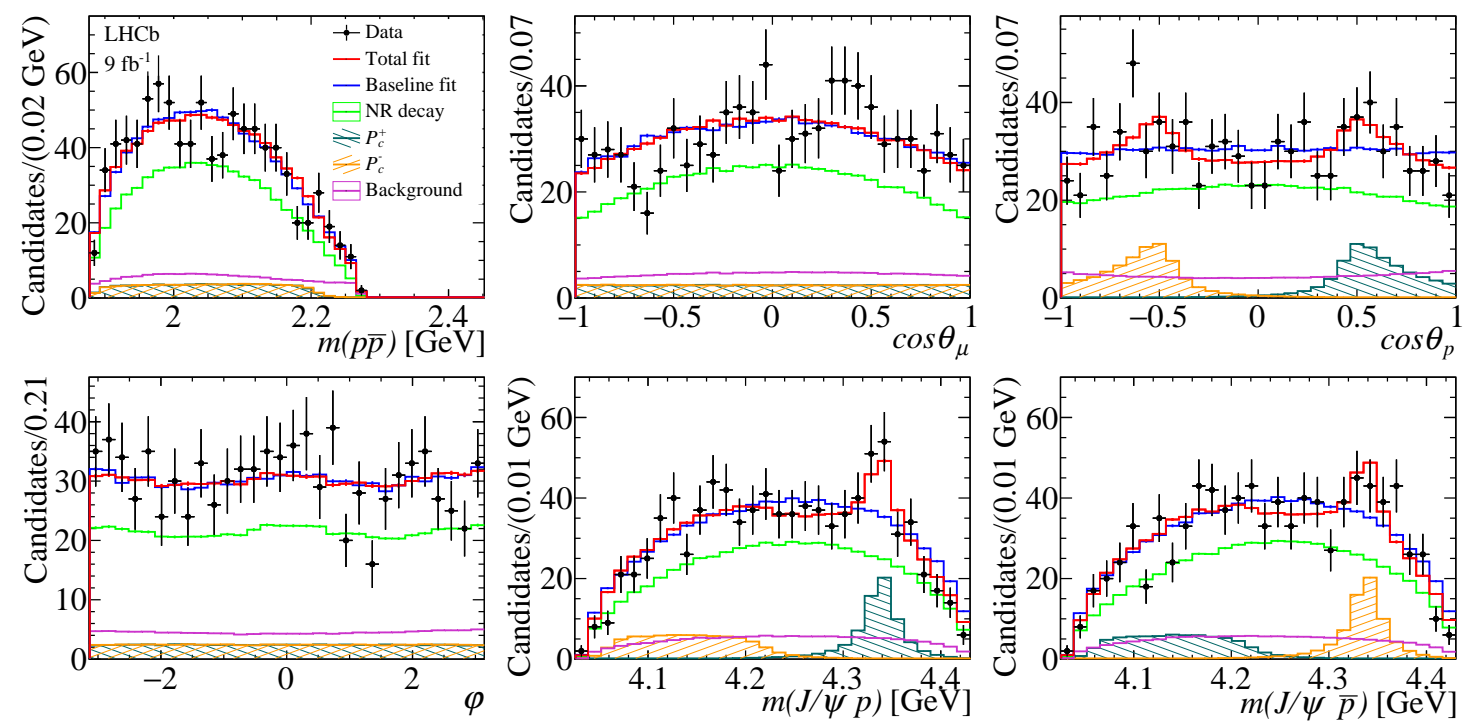

Figure 2: Projections of the angular $\left(\cos \theta_{\mu}, \cos \theta_{p}, \varphi\right)$ distributions, where $\theta_{\mu}, \theta_{p}$ are the helicity angles of the $\mu^{-}$and the $p$ in the $J / \psi$ and $X$ rest frame, respectively, and $\varphi$ is the azimuthal angle between the decay planes, together with the invariant-mass distributions $(m(p \bar{p}), m(J / \psi p), m(J / \psi \bar{p}))$. Results of the fit from the baseline model (without the $P_{c}$ state) are shown in blue and from the default model (with the $P_{c}$ state) in red.

LHCb dataset $\left(9 \mathrm{fb}^{-1}\right)$ is presented [11]. Thanks to an improved selection with a more sophisticated usage of machine learning algorithms, it was possible to improve the signal yield by a factor of 6 with respect to Run 1 (about 24,000 candidates) and obtain a better background rejection (4\% of background in a $\pm 15 \mathrm{MeV}$ window around the $B^{+}$peak). In the Dalitz plot shown in Fig. 3, four clear bands are present in the $J / \psi \phi$ invariant mass, corresponding to the $X$ states previously observed by LHCb with the Run 1 analysis, i.e. $X(4140), X(4274), X(4500)$ and $X(4700)$. In addition, a peak around $4 \mathrm{GeV}$ in the $J / \psi K^{+}$final state seems to suggest the existence of the $Z_{c S}^{+}$tetraquark. A full amplitude analysis in six dimensions is carried out. Since the amplitude model used in Run 1 does not describe entirely the invariant mass projections, as shown in the bottom row of Fig. 4, it is extended by adding two resonances decaying to the $J / \psi \phi$ final state, the $X(4630)$ and the $X(4685)$, 

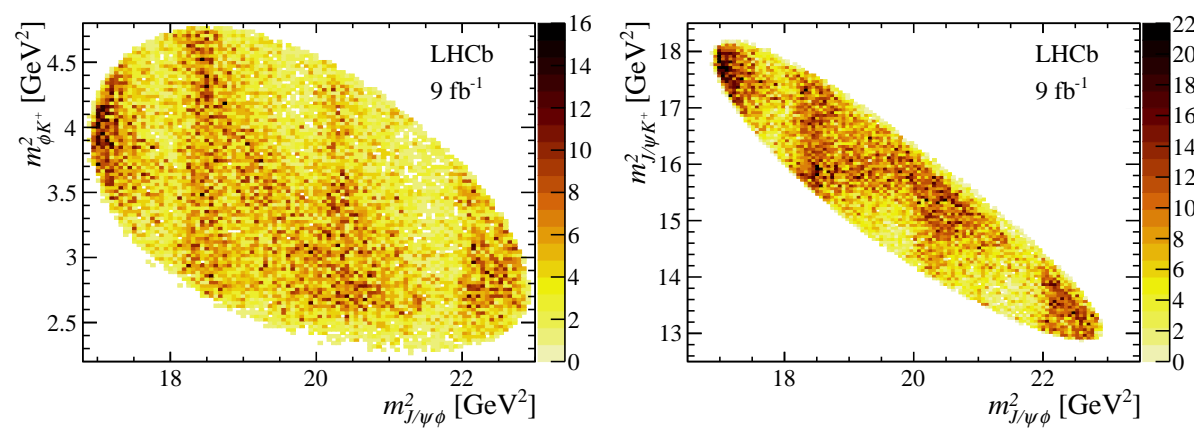

Figure 3: Dalitz plot of the $J / \psi \phi$ mass squared against the $\phi K^{+}$(left) and the $J / \psi K^{+}$(right) mass squared.

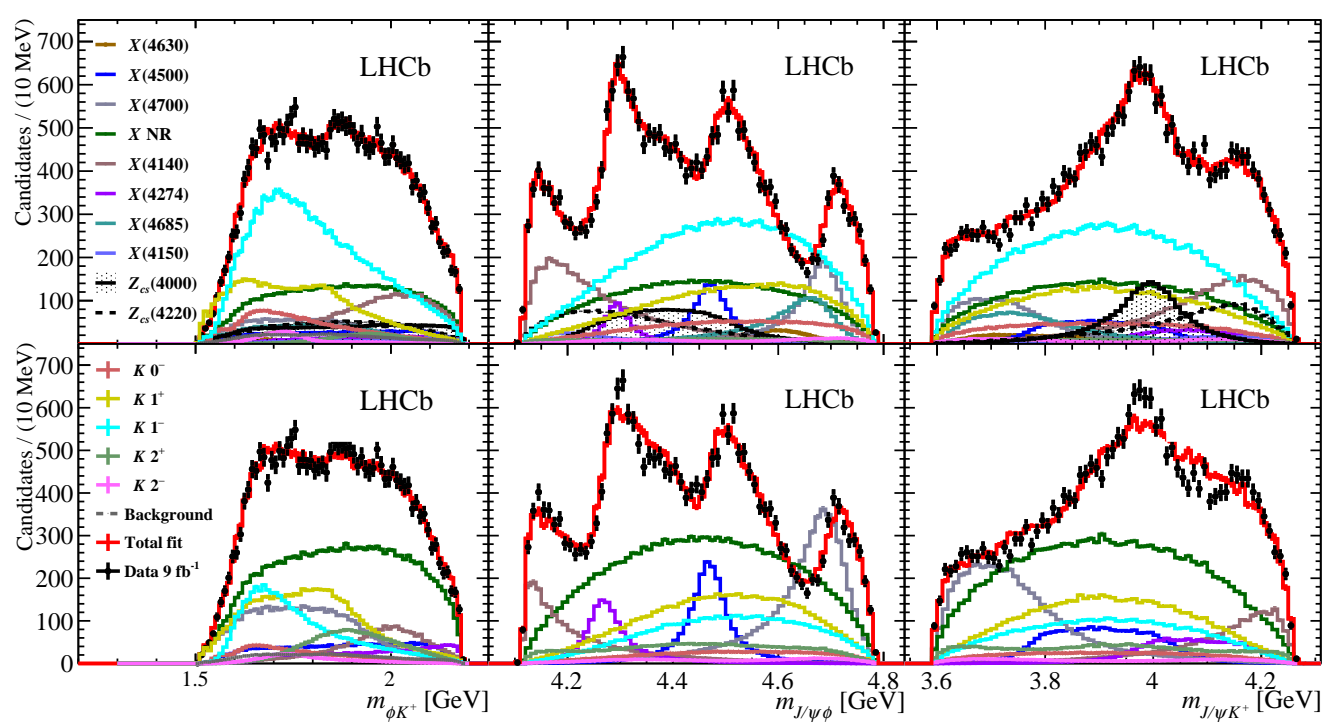

Figure 4: Projections over the $m\left(\phi K^{+}\right), m(J / \psi \phi)$ and $m\left(J / \psi K^{+}\right)$invariant masses of the amplitude fit performed with the Run 1 model only (bottom) and the full model (top).

and two resonances decaying to $J / \psi K^{+}$, the $Z_{c s}(4000)^{+}$and the $Z_{c s}(4220)^{+}$, which are candidates of tetraquarks with strangeness. All four states have been observed with a significance larger than $5 \sigma$. The quantum numbers have been determined for the $X(4685)$ and the $Z_{c s}(4000)^{+}$states to be $1^{+}$, while it is not possible to precisely assign them to the $X(4630)$ and the $Z_{c s}(4220)^{+}$states.

\section{Conclusion}

Promising results on tetra- and pentaquark spectroscopy have been presented, focusing on the analysis of $B_{s}^{0} \rightarrow J / \psi p \bar{p}$ decays, which reports the evidence for a new pentaquark-like state at mass $4337 \mathrm{MeV}$, and the analysis of the $B^{+} \rightarrow J / \psi \phi K^{+}$decays, with the observation of two new $Z_{c s}^{+}$ tetraquarks with strangeness and two new $X$ states. However, more exciting results are yet to come in the near future by analysing the current $\mathrm{LHCb}$ dataset or the larger one that will be available after the LHCb upgrade. 


\section{References}

[1] M. Gell-Mann, A schematic model of baryons and mesons, Phys. Lett. 8 (1964) 214.

[2] Zweig G., An SU3 model for strong interaction symmetry and its breaking, Version 2, CERNTH-412 (CERN) 1964.

[3] Belle Collaboration, S.K. Choi et al., Observation of a narrow charmonium-like state in exclusive $B^{ \pm} \rightarrow K^{ \pm} \pi^{+} \pi^{-} J / \psi$ decays, Phys. Rev. Lett. 91 (2003) 262001.

[4] LHCb Collaboration, R. Aaij et al., Observation of $J / \psi p$ resonances consistent with pentaquark states in $\Lambda_{b}^{0} \rightarrow J / \psi p K^{-}$decays, Phys. Rev. Lett. 115 (2015) 072001, arXiv: 1507.03414

[5] LHCb Collaboration, R. Aaij et al., Observation of a narrow pentaquark state, $P_{c}(4312)^{+}$, and of two-peak structure of the $P_{c}(4450)^{+}$, Phys. Rev. Lett. 122 (2019) 222001

[6] LHCb Collaboration, R. Aaij et al., Observation of $B_{(s)}^{0} \rightarrow J / \psi p \bar{p}$ decays and precision measurements of the $B_{(s)}^{0}$ masses, Phys. Rev. Lett. 122 (2019) 191804.

[7] Y. K. Hsiao and C. Q. Geng, $f_{J}(2220)$ and hadronic $\bar{B}_{s}^{0}$ decays, Eur. Phys. J. C75 (2015) 101.

[8] LHCb Collaboration, R. Aaij et al., Evidence for a new structure in the $J / \psi p$ and $J / \psi \bar{p}$ systems in $B_{s}^{0} \rightarrow J / \psi p \bar{p}$ decays, arXiv:2108.04720, submitted to Phys. Rev. Lett.

[9] S. U. Chung, Spin formalisms, 1971. CERN, Geneva, 1969 - 1970, doi: 10.5170/CERN1971-008

[10] LHCb Collaboration, R. Aaij et al., Observation of $J / \psi \phi$ structures consistent with exotic states from amplitude analysis of $B^{+} \rightarrow J / \psi \phi K^{+}$decays, Phys. Rev. Lett. 122 (2019), 222001.

[11] LHCb Collaboration, R. Aaij et al., Observation of new resonances decaying to $J / \psi K^{+}$and $J / \psi \phi$, Phys. Rev. Lett. 127 (2021) no. 8, 082001. 